\title{
PKN2 in colon cancer cells inhibits M2 phenotype polarization of tumor-associated macrophages via regulating DUSP6-Erk1/2 pathway
}

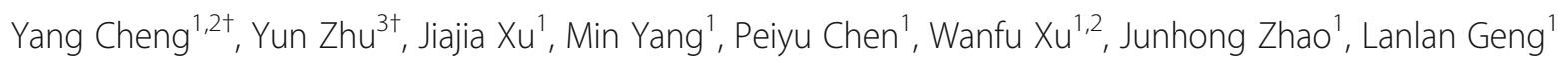
and Sitang Gong ${ }^{1 *}$

\begin{abstract}
Background: Protein kinase N2 (PKN2) is a PKC-related serine/threonine-protein kinase. PKN2 is required for tumor cell migration, invasion and apoptosis. However, the functional role of PKN2 in regulating tumor associated macrophages (TAMs) polarization in colon cancer has never been reported.

Methods: PKN2 expression in human colon cancer tissues was examined with immunohistochemistry (IHC). M1/M2 macrophage signatures were evaluated by RT-PCR, IHC and flow cytometry. The effects of PKN2 on tumor growth and TAM polarization were investigated both in vitro and in vivo. PKN2 targeted cytokines/pathway were analyzed by gene expression analysis and further confirmed by PCR, luciferase assay or western blot. Correlations between PKN2 and transcriptional factors for IL4 and IL10 were confirmed by ChIP-qPCR. The catalytic activities of PKN2 and DUSP6 were determined by kinase activity assay. Interactions between PKN2 and DUSP6 were confirmed by Co-IP.

Results: The expression of PKN2 in colon cancer cells predicted a favorable prognosis and was associated with low M2 macrophage content in human colon cancer tissues. PKN2 inhibited tumor growth in mice xenograft model and inhibited M2 phenotype polarization both in vitro and in vivo. Mechanistically, PKN2 suppresses the expression of IL 4 and IL 10 from colon cancer cells by inhibiting Erk1/2 phosphorylation, which is required for phosphorylation and binding of CREB and Elk-1 to the promoters of IL4 and IL10. DUSP6, which is phosphorylated and activated through direct association with PKN2, suppresses Erk1/2 activation.
\end{abstract}

Conclusions: The expression of PKN2 in colon cancer cells suppresses tumor associated M2 macrophage polarization and tumor growth. Targeting PKN2 signaling pathway may provide a potential therapeutic strategy for colon cancer.

Keywords: PKN2, Colon cancer, Macrophage polarization, Erk1/2, DUSP6

\section{Background}

Colon cancer is the third most common cancer worldwide, and the second most common cause of cancer-associated death $[1,2]$. Colon cancer arises from chronically inflamed tissues under the immune surveillance of tumor-infiltrating immune cells. Tumor associ-

\footnotetext{
*Correspondence: sitangg@yahoo.com

${ }^{\dagger}$ Equal contributors

'Digestive Department, Guangzhou Women and Children's Medical Center, Guangzhou Medical University, No.9 Jinsui Road, Guangzhou, Guangdong 510623, China

Full list of author information is available at the end of the article
}

ated macrophages (TAMs) affect many aspects of colon cancer, such as tumor angiogenesis and metastasis. TAMs display two main phenotypes, namely M1 and M2, which usually have contrasting effects on tumor progression [3]. M1 macrophages are the classically activated macrophages, which are polarized by lipopolysaccharide (LPS) and interferon- $\gamma$ (IFN- $\gamma)$. M1 macrophages express interleukin $-1 \beta$ (IL-1 $\beta), \quad$ IL-12 and cytotoxic substances such as inducible nitric oxide synthase (iNOS) [4]. M2 macrophages are alternatively activated macrophages, which are polarized in the presence of IL-4, IL-10 or IL-13. M2 macrophages express IL-10 
and IL-6, and angiogenic factors such as vascular endothelial growth factor (VEGF) [5]. High levels of M2 macrophages infiltration are associated with poor prognosis of colon cancer patients [6, 7]. Differentiation of TAMs to M1 or M2 phenotypes is regulated by the tumor microenvironment, including tumor cells [8-11]. Macrophages polarization is regulated by various microenvironmental signals derived from tumor cells. Tumor cells also secretes significant amounts of cytokines to induce the polarization of TAMs. Protein kinase N (PKN) represent a subfamily of protein kinase $\mathrm{C}$ (PKC). As one of the three PKN family members, protein kinase $\mathrm{N} 2$ (PKN2) was first described by Parker PJ et al. at 1994 [12]. PKN2 is a PKC-related serine/threonine-protein kinase and functions as effectors of Rho GTPases in diverse cellular pathways. PKN2 is required for cell cycle progression, cell migration, cell adhesion and transcription activation signaling processes $[13,14]$ and it plays important roles in tumor cell migration, invasion and apoptosis $[15,16]$. In HeLa cells, PKN2 has been reported to regulate mitotic entry and cytokinesis [17]. PKN2 regulates epithelial bladder cells speed and directmovement during cell migration and tumor cell invasion [18]. In human prostate cancer cells, PKN2 contributes to motility pathways and influences differentiation during prostate cancer progression [19]. PKN2 is highly expressed in triple-negative breast cancer cells and is required to support the growth of cancer [20]. However, in the intestine, the role of PKN2 in the regulation of tumor proliferation has never been reported, and the immunomodulatory effects of PKN2 have not been discussed.

In the present study, we found that PKN2 expression in colon cancer cells inhibited tumor growth by inhibiting TAM polarization to M2 like phenotype.

\section{Results}

\section{PKN2 correlates with better prognosis and low M2} macrophage content in human colon cancer

First, we examined the expression of PKN2 using a colon cancer tissue array containing colon cancer tissues from 90 patients. PKN2 expression was higher in the early stage of colon cancer (AJCC Cancer Staging Manual, 7th edition) (Tab.1). To quantify and distinguish the phenotype of the macrophages, the expression of iNOS

Table 1 PKN2 expression in tissues from colon cancer patients

\begin{tabular}{llllll}
\hline $\begin{array}{l}\text { AJCC } \\
\text { Cancer staging } \\
\text { manual }\end{array}$ & No. of patients & \multicolumn{4}{l}{ PKN2 expression } \\
\cline { 3 - 6 } & & & + & ++ & +++ \\
\hline I & 6 & $0(0.0 \%)$ & $0(0.0 \%)$ & $3(50.0 \%)$ & $3(50.0 \%)$ \\
III & 49 & $1(2.2 \%)$ & $11(22.4 \%)$ & $23(46.9 \%)$ & $14(28.5 \%)$ \\
IV & 34 & $2(5.8 \%)$ & $24(70.6 \%)$ & $8(23.5 \%)$ & $0(0.0 \%)$ \\
\hline
\end{tabular}

and CD86 (M1 marker), CD206 and CD163 (M2 markers) and CD68 (macrophage markers) were examined by IHC (Fig.1a). As shown in Fig.1b, the numbers of $\mathrm{CD} 8^{+}$cells were similar in colon cancer tissues of different AJCC stages but the numbers of CD206 ${ }^{+} /$ $\mathrm{CD}_{163^{+}}$cells were higher in late stage tumors and the number of $\mathrm{iNOS}^{+} / \mathrm{CD}^{2} 6^{+}$cells was higher in early stage tumors. We also explored PKN2 expression in normal colon, polyp, adenoma and metastatic adenocarcinoma. Higher expression of PKN2 was found in normal colon tissue compared with polyp, adenoma and metastatic adenocarcinoma. The expression of PKN2 decreased gradually in polyp, adenoma and metastatic adenocarcinoma (Fig. 1c and d). Additionally, we found that a higher PKN2 expression in the tumor tends to confer a significantly better prognosis (Fig. 1e). The number of $\mathrm{CD}^{+} 8^{+}$ cells was similar in both high $(++\&+++)$ and low $(-\&+)$ PKN2 expression tumor tissues. There were significantly more iNOS $^{+} / \mathrm{CD}^{+} 6^{+}$cells in high PKN2 expression tumor tissues, while the number of $\mathrm{CD} 206^{+} / \mathrm{CD} 163^{+}$ cells was significantly higher in low PKN2 expression tumor tissues (Fig. 1f). These findings indicated that PKN2 acts as a tumor suppressor in colon cancer and predicted a favorable prognosis. Additionally, PKN2 affected the differentiation of macrophages in human colon cancer tissue.

\section{PKN2 inhibits colon cancer growth and M2 macrophage polarization in vivo}

Our study showed that M2 macrophages promote while M1 macrophages inhibit the proliferation of the colon cancer cells in the co-culture system (Additional file 1: Figure S1).

To explore the effects of PKN2 on tumor proliferation and macrophage polarization, we generated HCT116 cells stably expressing wild-type PKN2 (PKN2-WT), dominant negative PKN2 (PKN2-K686R) and null vector (Vector) lentivirus. We found that the overexpression of PKN2-WT and PKN2-K686R did not affect colon cancer proliferation in vitro (Fig. 2a). Subsequently, a subcutaneous xenograft model of PKN2 transduced colon cancer cells in $\mathrm{BALB} / \mathrm{c}$ nude mice was constructed. Interestingly, we observed that the overexpression of PKN2-WT led to the suppression of tumor growth while PKN2-K686R led to the accelerated tumor growth in vivo (Fig. $2 \mathrm{~b}$ and c). The proliferation and apoptosis of xenograft tumor cells was detected by IHC staining of Ki67 and TUNEL assay. More Ki67-positive cells and less TUNEL-positive cells were observed in PKN2K686R group while less Ki67 positive cells and more TUNEL-positive cells were observed in the PKN2-WT group (Fig. 2d). Tumor cells and TAMs were separated from tumor tissues in mice by magnetic beads at the time of sacrifice (Fig. 2e). The expression of CD16/32 


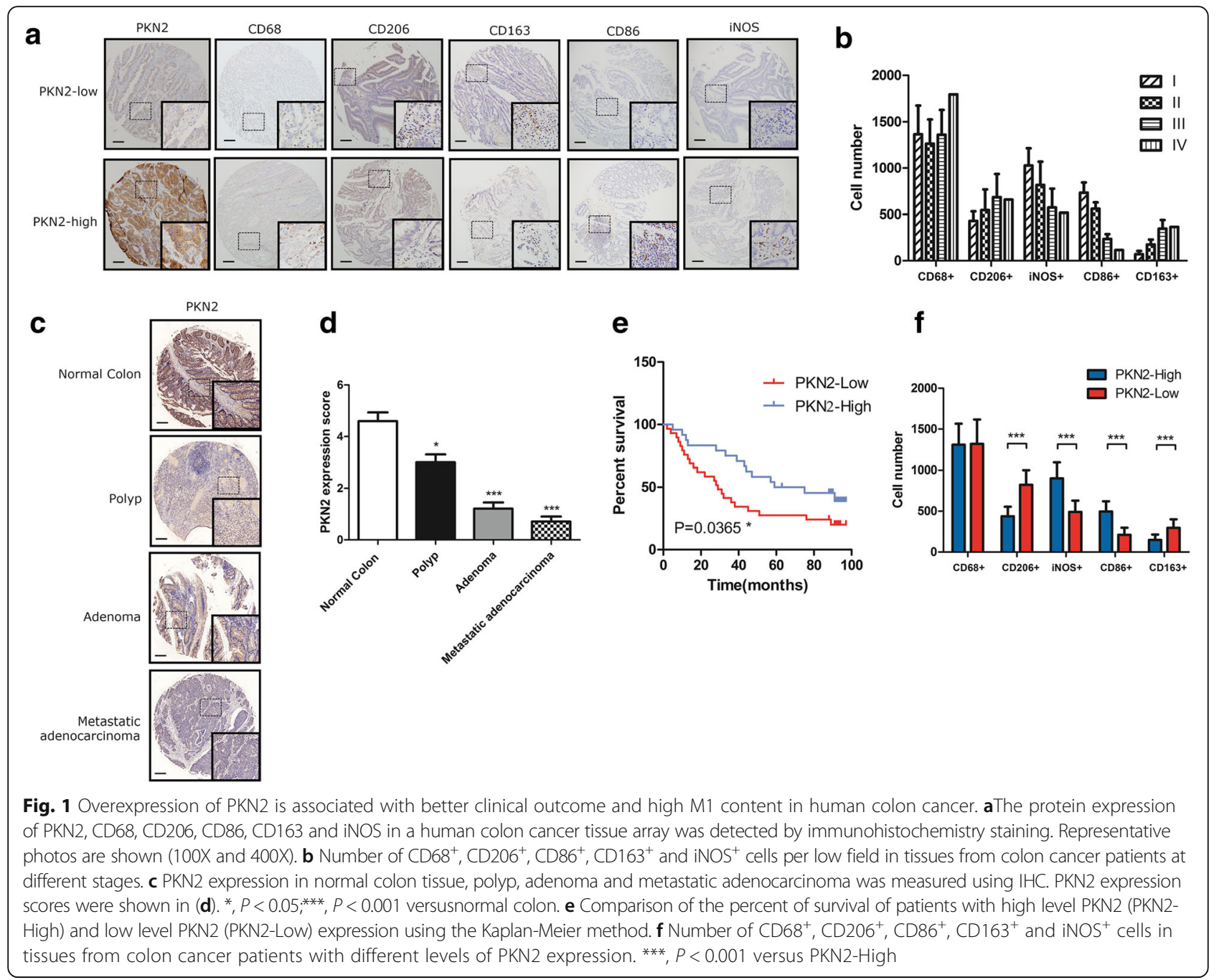

and CD206 was used to quantify M1 and M2 macrophages by flow cytometry. As shown in Fig.2f, PKN2K686R led to significantly increased $\mathrm{CD} 206^{+}$cells and decreased $\mathrm{CD} 16 / 32^{+}$cells in tumor tissues while ectopic expression of PKN2-WT led to decreased $\mathrm{CD}^{2} 26^{+}$cells and increased CD16/32 ${ }^{+}$cells. To further confirm the phenotype of these macrophages, the gene expression of typical M1 markers (Il1b, Tnf, Cxcl9, Il23, Ros1, Il12a and $I l 12 b$ ) and M2 markers (Tgfb1, Vegfa, Egf, Il6, Il10, Arg1, Retnla, and Ccl22) was investigated. A similar transcription pattern for macrophage markers was observed, as shown in Fig.2g \& $2 \mathrm{~h}$. Compared to the macrophages in WT group samples, the macrophages isolated from PKN2-WT tumor showed significantly higher expression of Illb, Tnf, Cxcl9, Il23, Ros1 and $I l 12 b$ and significantly lower expression of Tgfbl, Vegfa, Egf, Retnla and Il10, illustrating predominant M1 phenotype. In contrast, macrophages isolated from PKN2K686R tumors expressed significantly increased $T g f b 1$, Egf, Il10, Ccl22, Arg1 and decreased Il1b, Cxcl9, Il23,
Il12a, Il12b, indicating predominant M2 phenotype. These results suggested that while PKN2 did not alter colon cancer proliferation in vitro, it inhibited the growth of colon cancer cells in vivo, most likely associated with the increased differentiation of M1 and decreased differentiation of M2 macrophages.

\section{Colon Cancer specific PKN2 expression promotes M1 macrophage polarization in vitro}

We next evaluated how colon cancer specific PKN2 expression may promote M1 polarization in vitro. Cardiolipin, as the natural activator of PKNs [21], was used to stimulate colon cancer cells. Cardiolipin stimulation did not alter the proliferation or colony formation of colon cancer cells (Additional file 1: Fig.S2 a-c). However, cardiolipin treated HCT116 cells significantly decreased M2 macrophage polarization in vitro (Additional file 1 : Fig.S2 d\&e). Colon cancer cells stably overexpressing wild-type PKN2 or shRNA-PKN2 were generated (Fig. 3a, d, and g). Human CD14 ${ }^{+}$monocytes were co- 


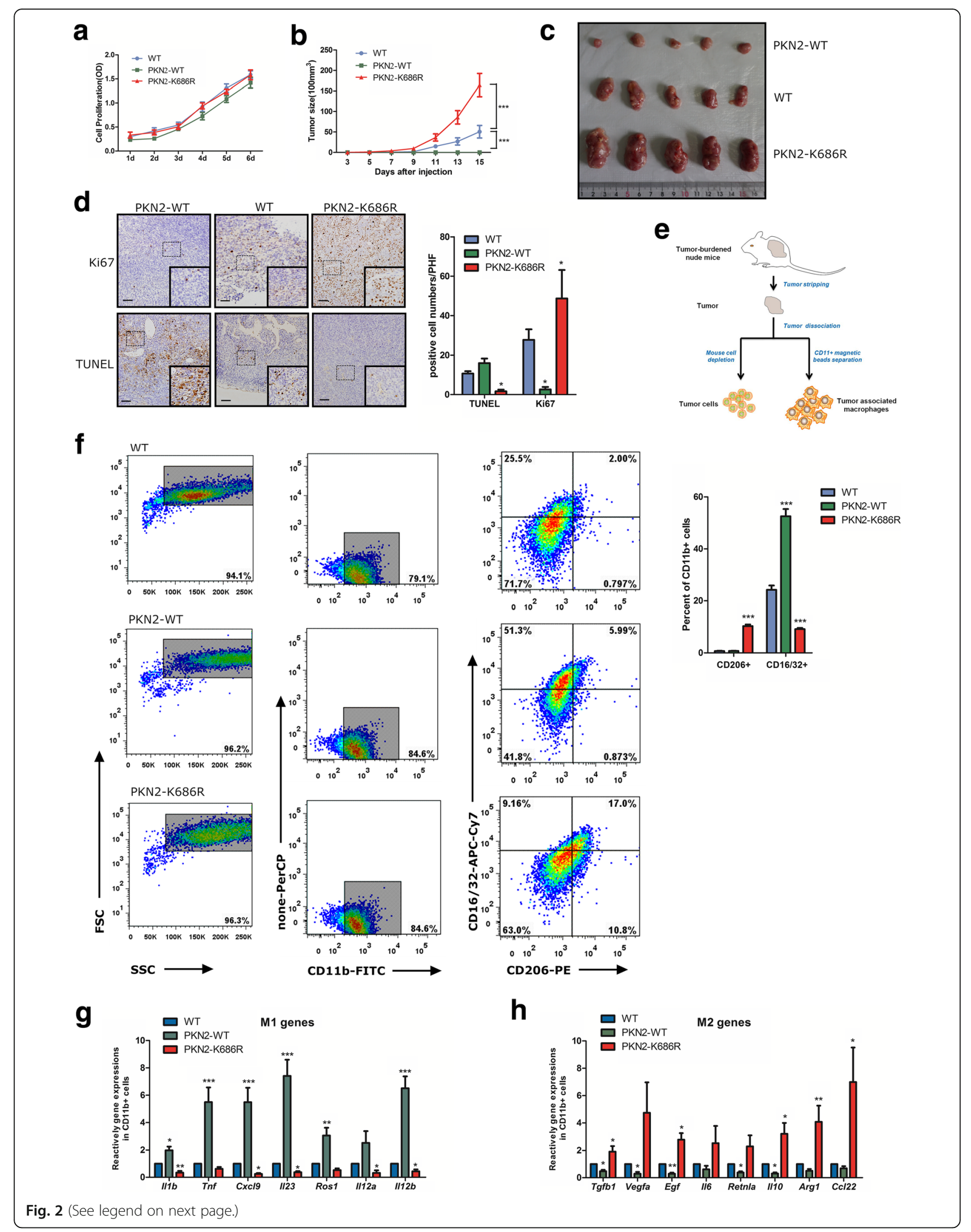


(See figure on previous page.)

Fig. 2 PKN2expression inhibits tumorformationand decreased macrophages polarizing towards the M2 type in the xenograft tumor. a HCT116 cells were stably infected with vector (WT), PKN2-WT, PKN2-K686R lentivirus and cultured for 1-6 days. Cell proliferation was detected by CCK8 assay. $\mathbf{b}$ Tumorigenesis assay of Balb/c nude mice subcutaneously injected with PKN2-WT/PKN2-K686R transduced or wild-type HCT116cells $(n=10)$. ***, $\mathrm{P}<0.001$ versus WT. $\mathbf{c}$ Representative photos of tumors from mice of the various groups. $\mathbf{d}$ TUNEL assay \&lHC staining of Ki67 in tumor tissues in mice xenograft model and positive cell numbers per high field were counted. ${ }^{*}, P<0.05$ versus WT. e Schematic picture on the procedure for separation of Tumor cells \&TAM. $\mathbf{f} C D 11 b^{+}$macrophages were separated from murine tumor tissues using CD11b magnetic beads. Surface expression of CD16/32 and CD206 was detected in CD11 b macrophages usingflow cytometry. The percent of CD16/32 $2^{+}$or CD206 ${ }^{+}$cells in CD11 b ${ }^{+}$macrophages were assayed..**, $P<0.001$ versus WT. g Relative gene expression of M1 marker (II1b, Tnf,Cxc19, II23, Ros1,II12a, and II12b) and M2 marker(Tgfb1, Vegfa, Egf, II6, Retnla, II10, Arg1 and Cc122) (h) in the tumor tissues of mice.*, $P<0.05$; ${ }^{* *}, P<0.01$; ***, $P<0.001$ versus WT

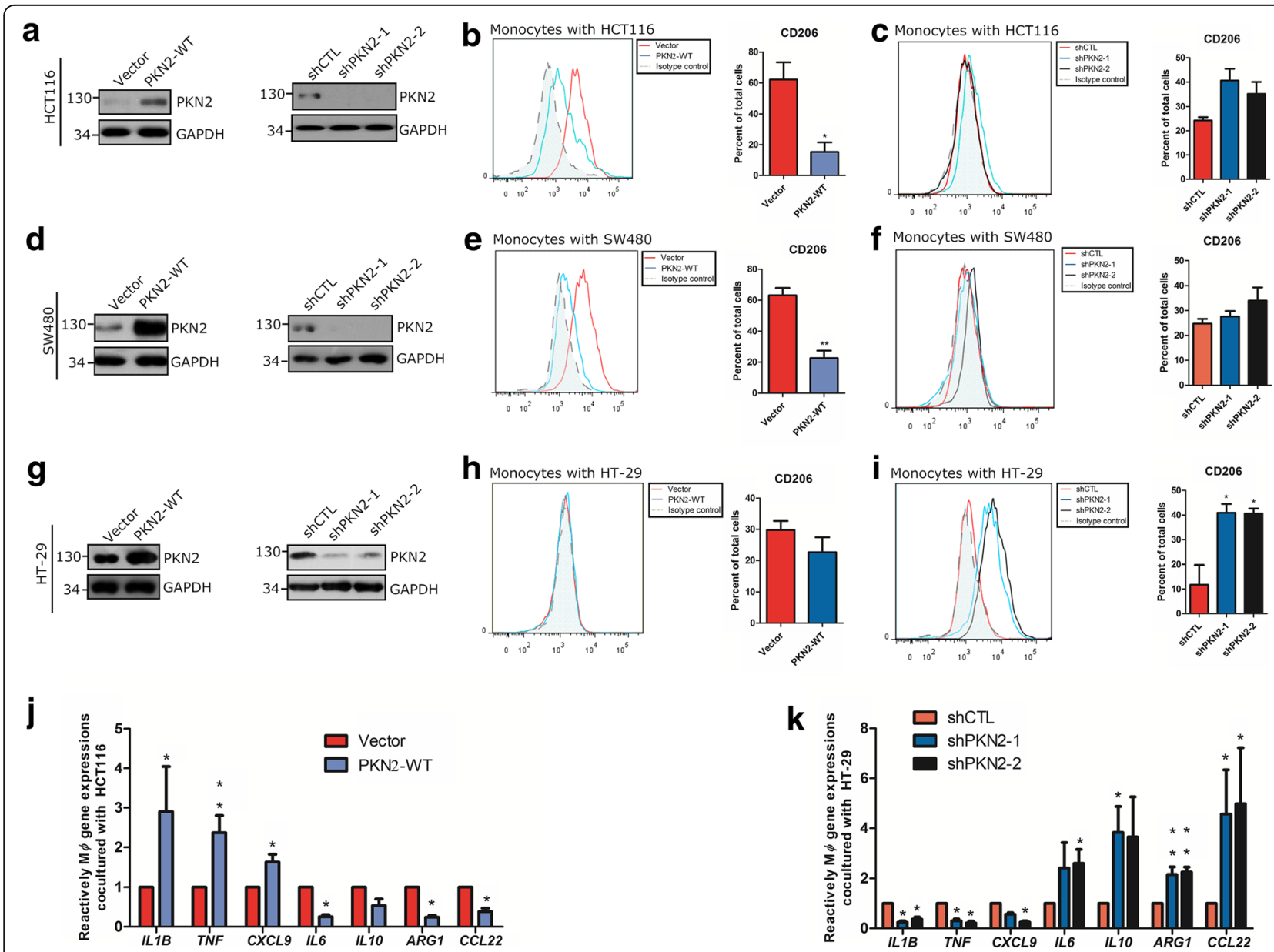

Fig. 3 PKN2 in colon cancer cells induces the differentiation of monocytes into M2 polarized macrophages in vitro. a HCT116

cells were stably transfected with vector/PKN2-WT or shCTL/ shPKN2-1/ shPKN2-2. The protein expression of PKN2 was detected by western blotting in total protein extracts. Human CD14 ${ }^{+}$monocytes were separated from peripheral blood and co-cultured with vector/PKN2-WT (b) or shCTL/ shPKN2-1/ shPKN2-2 (c) transfected HCT116 for 4 days, respectively. E:T ratios for colon cancer cells to monocytes were 50:1. Flow cytometry was used to explore surface expression of CD206. ${ }^{*}, P<0.05$ versus Vector. (d-f) SW480 cells were treated and co-cultured with CD $14^{+}$monocytes as indicated in (a-c). Western blotting was used to evaluate PKN2 expression in SW480 cells. Flow cytometry was used to explore surface expression of CD206 in differentiated macrophages. ${ }^{*}, P<0.01$ versus Vector. (g) $\sim$ (i) $H$ T-29 cells were treated and co-cultured with CD14 ${ }^{+}$monocytes as indicated in (a) (c). PKN2 and CD206 expression was analyzed. *, $P<0.05$ versus shCTL. (j) CD14 ${ }^{+}$monocytes were treated as indicated in (b), gene expression of ILIB, TNF, CXCL9, IL6, IL10, ARG1 and CCL22 in monocytes was detected. (k) CD14+ monocytes were treated as indicated in (i), gene expression of IL1B, TNF, CXCL9, IL6, IL10, ARG land CCL22 in monocytes was detected. *, $P<0.05 ; * *, P<0.01$ versus Vector or shCTL 
cultured with treated colon cancer cells. The numbers of $\mathrm{CD}_{206}{ }^{+}$(M2) macrophages differentiated from CD14 ${ }^{+}$ peripheral blood mononuclear cells (PBMCs) were dependent on effector: target (E:T) ratios, and E:T ratio of 50:1 had the best inductive effects (Additional file 1: Figure S3). The overexpression of wild-type PKN2 (PKN2WT) in HCT116 and SW480 cells decreased the number of $\mathrm{CD}_{206^{+}}$macrophages that differentiated from CD14 ${ }^{+}$ monocytes (Fig. 3b and e). HT-29 cells preinfected with shPKN2 significantly increased the number of CD206 ${ }^{+}$ macrophages (Fig. 3i). Similar patterns for macrophage markers were observed using RT-PCR (Fig. 3j and k). Moreover, macrophages cocultured with low level PKN2 cancer cells promoted, while macrophages polarized by high level PKN2 cancer cells inhibited colon cancer cells proliferation by arresting the cell cycle and increasing apoptosis (Additional file 1: Fig.S4). These results further supported the hypothesis that PKN2 expression in colon cancer cells inhibits macrophage differentiation into the M2-like phenotype in the colon cancer cell milieu and consequently inhibits tumor growth.

\section{PKN2 suppresses M2 polarization by inhibiting colon cancer cell expression of IL4 and IL10}

M2 macrophage differentiation relies on the presence of IL10 or IL4. We hypothesized that PKN2 might suppress M2 polarization via altering the expression profiles of inflammatory cytokines of colon cancer cells. To this end, we analyzed the differentially expressed genes in WT, PKN2-WT and PKN2-K686R tumor cells separated from xenograft as shown in Fig. 2e using gene expression profiles analysis. As shown in Fig. 4a, in KEGG 'chemokine signaling pathway' and 'cytokine-cytokine receptor interaction pathway' analyses, 17 genes were significantly upregulated in PKN2-K686R cells but downregulated in PKN2-WT cells, indicating that these genes are negatively regulated by PKN2. Among these genes, two cytokines IL4 and IL10 were identified (Fig. 4b). We further explored the relationship between PKN2 and these two cytokines both in vivo and in vitro. The mRNA levels of IL4 and IL10 in tumor cells separated from different xenografts were detected. The mRNA level of IL4 and IL1O was significantly decreased in PKN2-WT tumor cells, but increased in PKN2-K686R tumor cells, indicating that IL4 and IL10 are negatively regulated by PKN2 (Fig. 4c). We also detected the cytokine levels in the culture supernatants of PKN2-depleted HT-29 cells, and PKN2WT ectopically overexpressed SW480 and HCT116 cells. Significantly decreased IL4 and IL10 levels were found in PKN2 overexpression colon cancer cells, while profoundly increased IL4 and IL10 expression was detected in PKN2-depleted cells (Fig. 4d). Moreover, cardiolipin treated HT-29 cells secreted lower levels of IL4 and IL10 in vitro (Additional file 1: Figure S2 f\&g). The promoter activities of ILIO and IL4 were decreased in PKN2 overexpressed SW480 cells but markedly increased in PKN2-depleted cells as shown in luciferase reporter assays (Fig. 4e). Rescue studies showed that neutralizing antibodies of IL4 and IL10 attenuated the upregulated level of $\mathrm{CD}_{206}{ }^{+}$macrophages induced by PKN2-depleted HT-29 cells. Moreover, neutralizing antibodies of IL4 and IL10 reduced the upregulated CD86 ${ }^{+}$ macrophages induced by overexpressed PKN2 in HCT116 cells (Fig. 4f and g). These results supported that PKN2 reduced macrophage polarization to the M2like phenotype via decreasing the expression and secretion of IL4 and IL10.

\section{PKN2 modulates IL4 and IL10 expression via negative regulation of Erk1/2}

We showed that PKN2 targets and regulates IL4 and IL10 at the transcriptional level. Next, we attempted to elucidate the regulatory mechanisms of PKN2 mediated cytokine expression. KEGG pathway analysis showed that the 'MAPK signaling pathway' (KEGG No.4010) was the most relevant pathway (Additional file 1: Table S1). The heatmap analysis of the differentially expressed genes regulated by PKN2 is shown in Additional file 1: Figure S5. To confirm the role of PKN2 in the MAPK pathway, different doses of HA-tagged PKN2-WT vectors were transfected into RKO cells. Western blot analysis showed that PKN2 induced a dose-dependent decrease in the phosphorylation of Erk1/2 (Thr202/ Tyr204) but had no effect on the phosphorylation of p38, JNK and Erk5 (Fig. 5a). Fig 5b showed that knocking down PKN2 or overexpressing dominant negative PKN2 upregulated the phosphorylation of Erk1/2 (pErk1/2). Moreover, IHC staining showed that p-Erk1/2 was upregulated in PKN2-WT overexpressed murine tumor tissue. Additionally, there was a strong negative relation between PKN2 and p-Erk1/2 expression levels in murine tumor tissue (Fig. 5c). However, the expression of Erk1/2 did not show a significant difference between the groups (data not shown). Subsequently, we explored whether PKN2 mediates the transcription of IL4 and IL10 via inhibition of the Erk1/2 pathway. HT29 was stably transfected with shPKN2 or shCTL and treated with Erk1/2 inhibitors (U0126 or SCH772984). The potential signal cascade, including the activation of Erk $1 / 2$ by phosphorylation and the expression of IL4 and IL10, was examined (Fig.5d and e). As shown in Fig. 5 (d and e; 1 vs. 4 ), the downregulation of PKN2 by shRNA enhanced the phosphorylation of Erk $1 / 2$ but decreased the expression of IL4 and IL10. Treatment with U0126 and SCH772984 significantly abrogated the phosphorylation of Erk1/2 and markedly reduced the expression of 


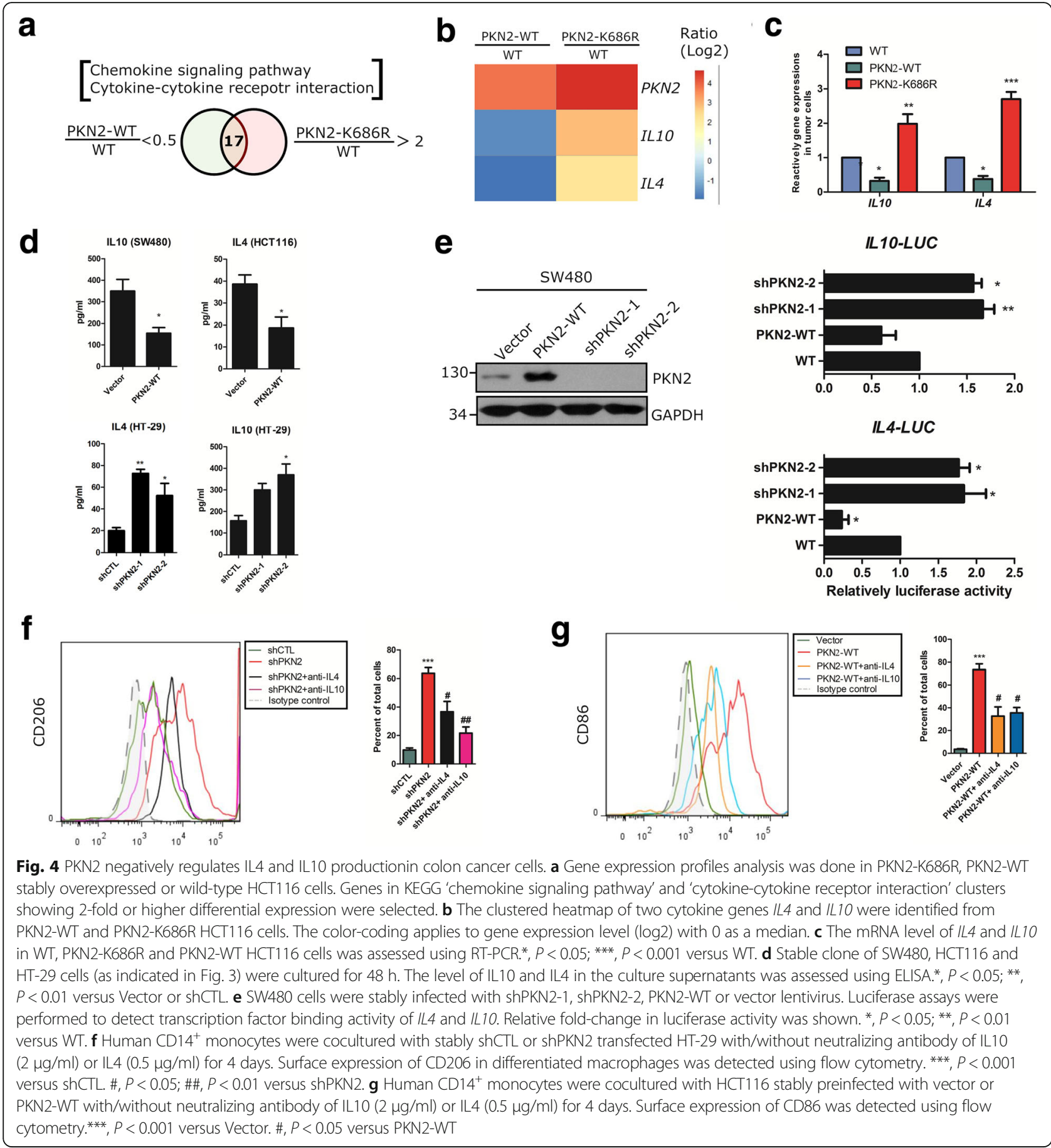

IL4 and IL10 by knocking down PKN2 (Fig. $5 \mathrm{~d}$ and e; 1 vs. 2,$3 ; 4$ vs. 5,6 ). $\mathrm{CD}^{+} 4^{+}$monocytes were cultured with HT29 cells stably transfected with shCTL or shPKN2. The knockdown of PKN2 increased the number of $\mathrm{CD}^{206^{+}}$ macrophages but decreased the number of $\mathrm{CD} 86^{+}$macrophages, and SCH772984 could partially abolish these effects (Fig. 5f). These results further confirmed that PKN2 suppresses IL4 and IL10 expression through the inhibition of Erk1/2 phosphorylation.
PKN2 inhibits DNA binding ability of CREB and Elk-1 to the promoter of IL4 and ILI0

To elucidate the transcriptional factors involved in PKN2 mediated IL4 and IL10 expression, transcription factor (TF) activity arrays were performed in shCTL/ shPKN2 transfected HT-29 cells treated with U0126 or solvent. As shown in Additional file 1: Figure S6 a, CREB (red) and Elk-1 (blue) were two obvious differentially expressed TFs. The relative level of CREB and Elk- 

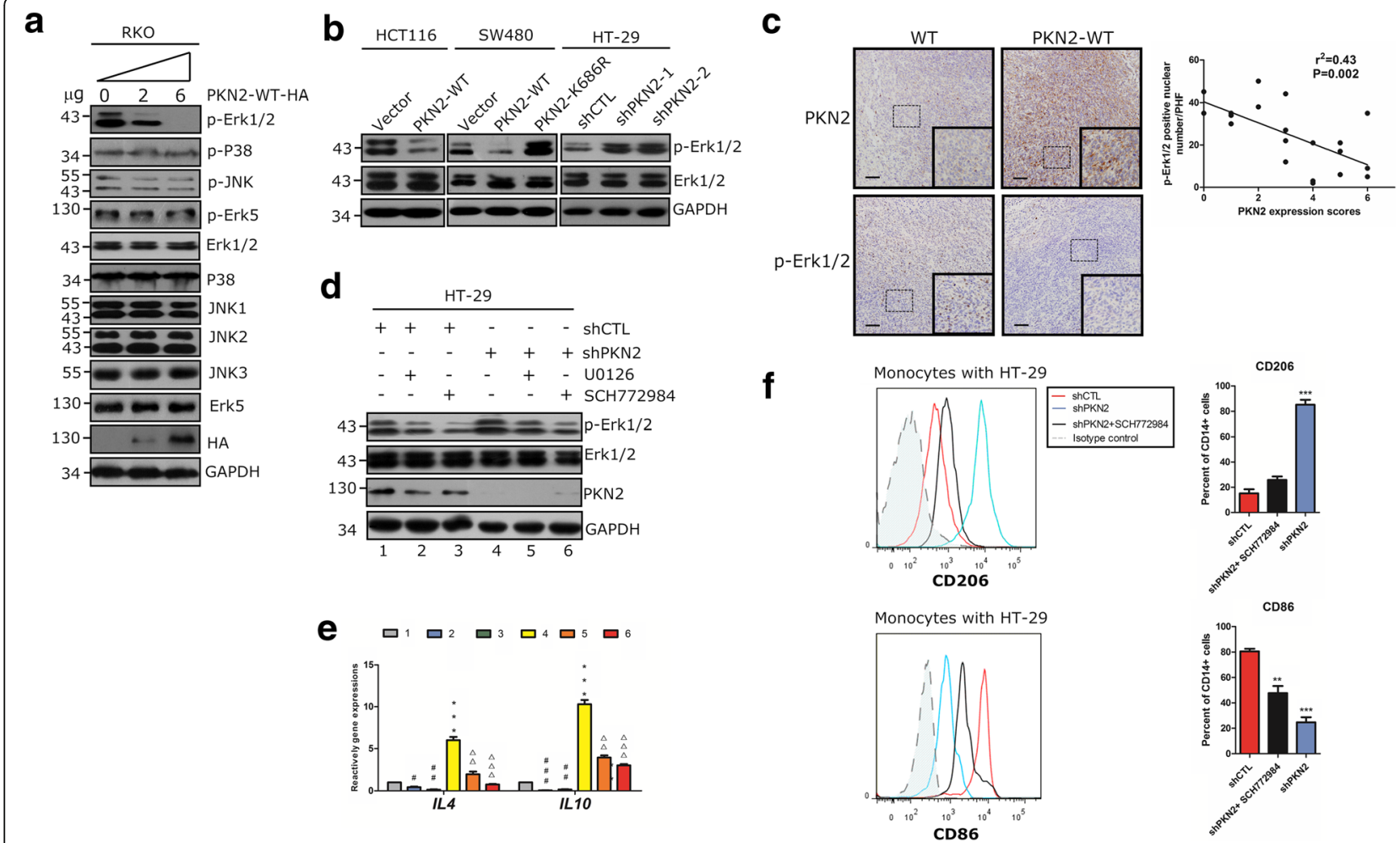

Fig. 5 PKN2 negatively regulates Erk1/2. a RKO cells were transfected with 0, 3 or $6 \mu \mathrm{g}$ PKN2-WT-HA.Western blotting was used to detect the indicated proteins. b Stable clones of SW480, HCT116 and HT-29 cells (as indicated in Fig. 3) were detected for the expression of p-Erk1/2, Erk1/2 and GAPDH using western blotting. $\mathbf{c} I H C$ staining of PKN2 and p-ERK1/2 in the tumor tissues of mice xenograftmodels. The correlation between p-Erk1/2 positive number per high field and the PKN2 expression score was explored. $\mathbf{d}$ HT-29cells were stably transfected with shCTL or shPKN2

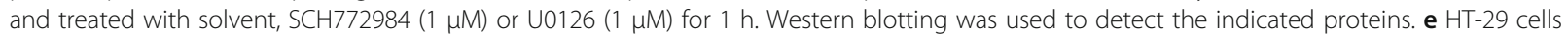
were treated as indicated in (d). IL4 and IL10 gene expression was detected by RT-PCR.***, $P<0.001$ versus lane 1. \#, $P<0.05$; \#\#, $P<0.01$; \#\#\#, $P<0.001$ versus lane 1. $\Delta \Delta, P<0.01 ; \Delta \Delta \Delta, P<0.001$ versus lane4. $\mathbf{f}$ Human $C D 14^{+}$monocytes were cocultured with stably transfected shCTL or shPKN2 HT-29 cells treated with SCH772984 (50 nM) or solvent for 4 days. Surface expression of CD206 and CD86 in differentiated macrophages was detected using flow cytometry.**, $P<0.01 ;{ }^{* * *}, P<0.001$ versus shCTL

1 activities were shown in Additional file 1: Figure S6 b. The phosphorylation of CREB and Elk-1 was detected to confirm the activation of the two TFs. The phosphorylation of CREB (Ser133) and Elk-1 (Ser383) was elevated by knocking down PKN2, but suppressed by U0126 treatment in HT-29 cells (Fig. 6a). Moreover, the overexpression of PKN2 in SW480 and HCT116 cells reduced the levels of p-CREB and p-Elk-1 (Fig. 6b). Flagtagged wild-type Elk-1 and CREB and HA-tagged wildtype PKN2 were transfected into RKO cells, respectively (Fig. 6c). The RT-PCR results showed that PKN2 overexpression reduced the gene expression of IL4 and IL10, while the transfection of CREB and Elk-1 could partly rescue this reduction. Using the JASPAR and PROMO databases, we identified the potential binding sites of CREB and Elk-1 in the promoters of IL4 and IL1O $(-1000 \mathrm{~kb} \sim+1 \mathrm{~kb})$ (Fig. 6d). ChIP assays were performed to confirm the effects of PKN2 on the binding between CREB/Elk-1 and the promoters of IL4 and IL1O. As shown in Fig. 6e, both CREB and Elk-1 bound to the promoter of IL10, but only Elk-1 could bind to the promoter of IL4. PKN2 overexpression obviously decreased the DNA binding capacity of CREB and Elk-1 but reduced PKN2 expression significantly increased the DNA binding of the two TFs. These data suggested that PKN2 inactivated the transcription of IL4 and IL1O via inhibition of the binding ability of Elk-1 and CREB to the promoter.

\section{PKN2 reduced Erk1/2 phosphorylation via directly binding and activation of DUSP6}

Next, we explored the underlying mechanism involved in the negative regulation of $\mathrm{p}$-Erk1/2 by PKN2. Dual specificity phosphatase 6 (DUSP6) was considered an inhibitor of Erk1/2 [22]. DUSP6 expression in human colon cancer tissues was evaluated by IHC. As shown in Fig. 7a, there was a significantly positive relation between PKN2 and DUSP6 expression in human colon cancer tissues. Survival analysis showed that the survival time of the PKN2-High \& DUSP6-High groups was 

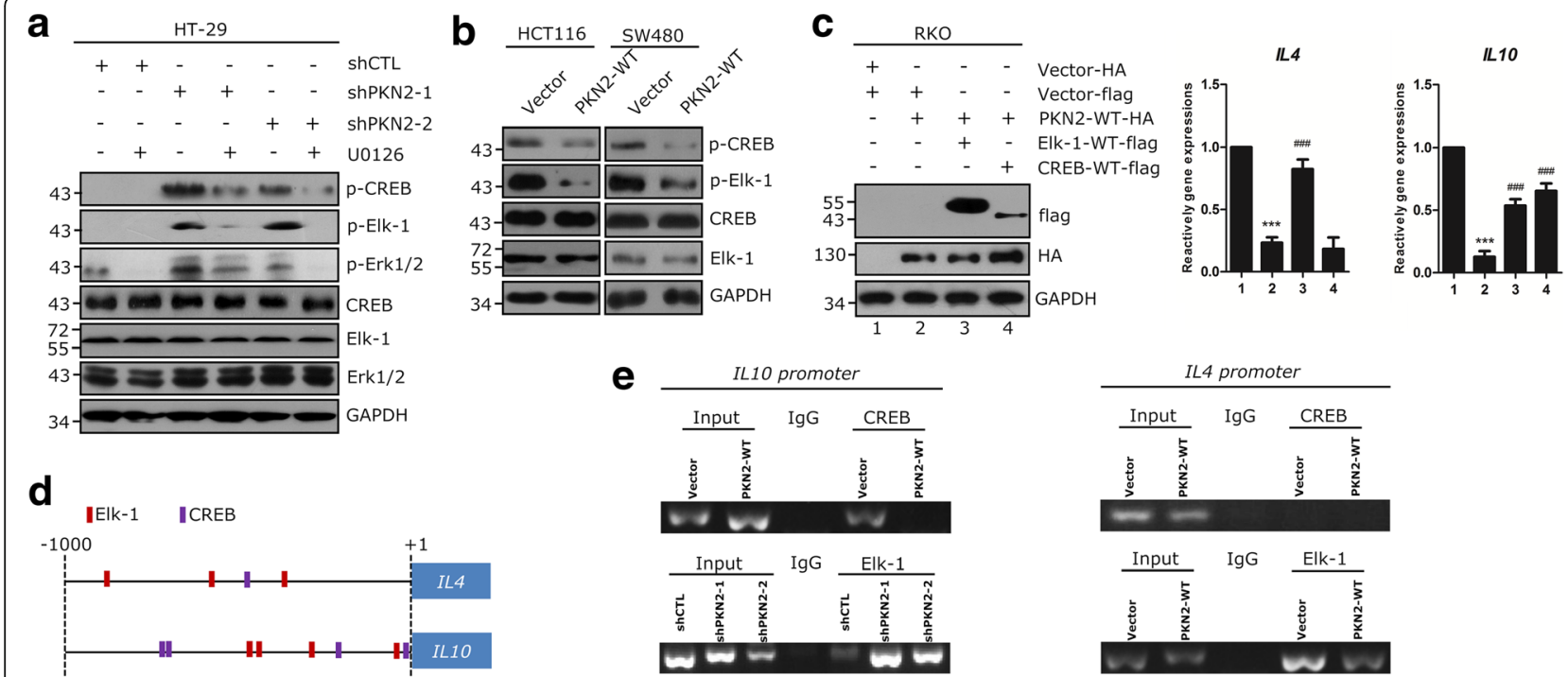

Fig. 6 PKN2 inhibits the transcriptional activities of CREB and Elk-1. a HT-29cells were stably transfected with shCTL, shPKN2-1 or shPKN2-2 and treated with solvent or U0126 (1 $\mathrm{MM}$ ) for $1 \mathrm{~h}$. Western blotting was used to detect the indicated proteins. b Stable clones of SW480, HCT116 were detected for indicated proteins using western blotting. c RKO cells were transfected with PKN2-WT-HA, CREB-WT-flag, Elk-1-WT-flag or control vectors. IL4 and IL10 gene expression was detected. ${ }^{* *}, P<0.001$ versus lane 1. \#\#\#, $P<0.001$ versus lane 2. d Diagram shows the predicted binding sites of Elk-1 and CREB on the $-1000 \mathrm{bp} \sim+1$ bp promoter regions of IL 4 and IL10. e ChIP assays of Elk-1 or CREB on the chemokine promoters in SW480 stably transduced with PKN2-WT or vector, and HT-29 cells transduced with shCTL or depletion of PKN2. These assays were repeated three times, and representative photos are shown

obviously higher than that of the PKN2-Low \& DUSP6Low groups (Fig. 7b). Additionally, knocking down DUSP6 could rescue the level of p-Erk1/2 in response to PKN2-WT overexpression (Fig. 7c). The phosphatase activity assays showed that PKN2 increased the activity of DUSP6 in colon cancer cell lines (Fig. 7d). Cardiolipin elevates the autophosphorylation and promotes the catalytic activity of PKN2 [23]. Thus, we used cardiolipin as an activator of PKN2 in PKN2-WT or PKN2-DN transduced 293 T cells. As shown in Fig. 7e, the activity of DUSP6 significantly increased in $293 \mathrm{~T}$ cells transfected with PKN2-WT (lane 5 vs. lane 3; lane 6 vs. lane 4), while the transfection of PKN2-K686R did not elevate DUSP6 activity (lane 7 vs. lane 3; lane 8 vs. lane 4). Coimmunoprecipitation(Co-IP) assay showed that DUSP6 directly interacted with PKN2 in colon cancer cell lines (Fig. 7f). Thus, we transfected wild-type DUSP6-flag protein with or without different amounts of HAtagged PKN2-WT in 293 T cells. As shown in Fig. 7g, PKN2 directly bound to DUSP6 in a dose-dependent manner. Domain-truncation experiments showed that PKN2 potentially binds to the linker region of DUSP6 (aa 150-205) (Fig. 7h and i). These data suggested that PKN2 inhibited the activity of Erk1/2 by directly binding to DUSP6.

\section{Discussion and conclusions}

PKN2 has been recognized as a regulator of multiple aspects of cellular events, such as cell cycle progression, cell migration, and cell adhesion. Recently, PKN2 has emerged as a regulator of cancer growth, invasion and metastasis $[19,20]$. However, the role of PKN2 in colon cancer has never been explored. In the present study, we provided the first evidence that low PKN2 expression is strongly correlated with advanced colon cancer and poor prognosis. These results support the notion of PKN2 as a potential tumor suppressor in colon cancer. A previous study reported that PKN2 could promote cell proliferation [17]. The present study showed that the overexpression or activation of PKN2 had no influence on the proliferation of colon cancer cells in vitro. Interestingly, in a mice xenograft model, PKN2 significantly inhibited tumor growth. Based on these observations, we hypothesized that PKN2 signaling inhibits the proliferation of tumor cells by modulating the reconstitution of the tumor microenvironment rather than acting directly on tumor cells.

Emerging evidence has revealed that TAMs are associated with tumor growth, invasion and metastasis [24]. M2- polarized TAMs promote tumor growth and invasion, while M1-like polarized TAMs act as tumor suppressers. In several human cancers, a higher density of the M2 macrophages and lower density of the M1 macrophages is associated with worse clinical outcomes. Consistent with the previous analysis, the present study demonstrated that M2 macrophages promote while M1 macrophages inhibit proliferation of the colon cancer cells. Moreover, we demonstrated that macrophages 


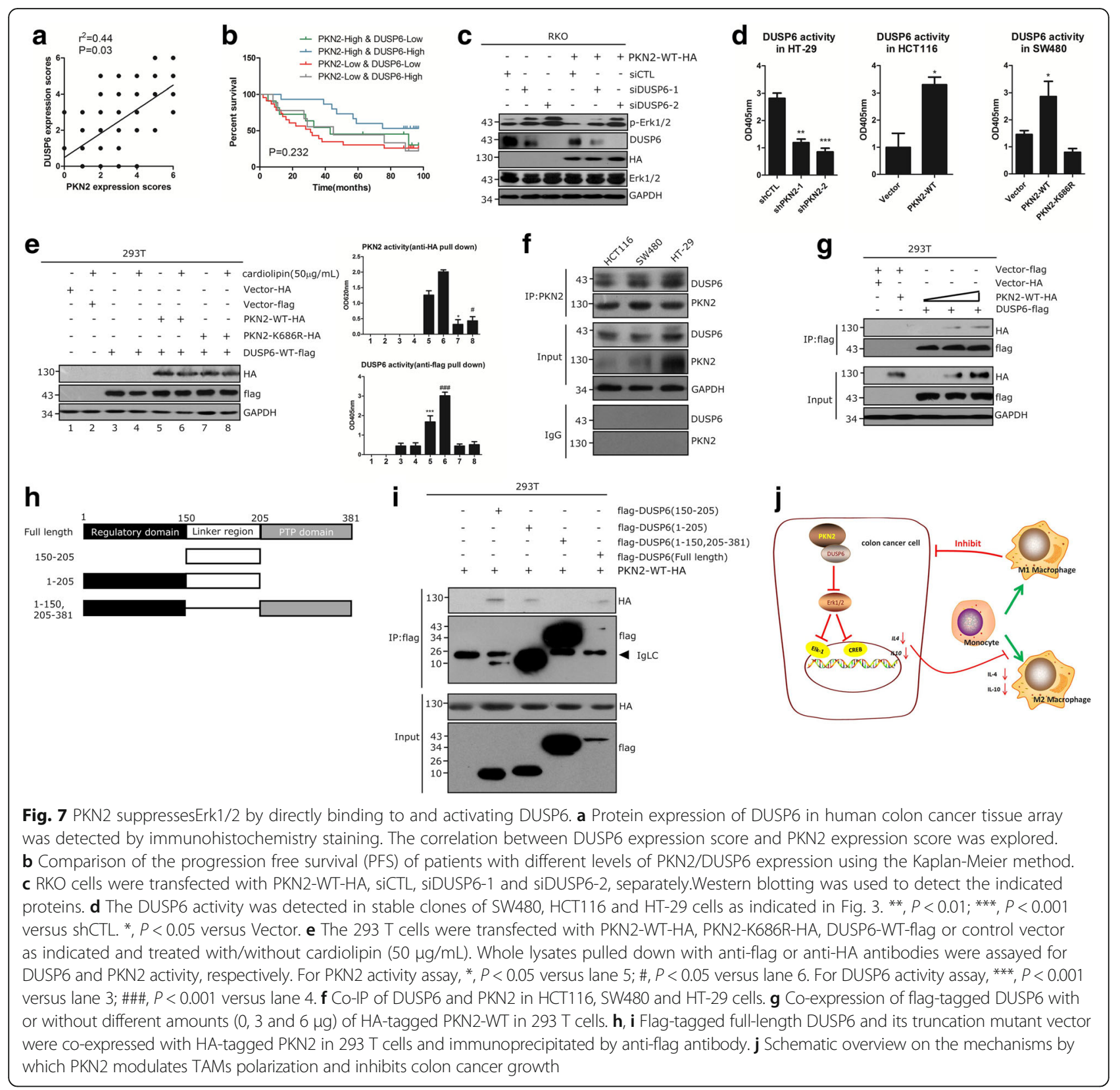

cocultured with low level PKN2 cancer cells promote, while macrophages polarized by high level PKN2 cancer cells inhibit colon cancer cell proliferation.

Most TAMs exhibit an M2- phenotype [25]. Macrophage polarization within tumor tissues is regulated by various microenvironmental signals derived from tumor cells $[26,27]$. We provided the first evidence that the number of M2-type TAMs in clinical colon cancer tumor tissues was negatively correlated with PKN2 expression in tumor cells. This finding prompted us to investigate whether the expression of PKN2 in colon cancer cells can mediate macrophage polarization. We demonstrated that PKN2 expression in colon cancer cells inhibits M2-like polarization of TAMs both in vitro and in vivo. Wild-type PKN2 overexpression in colon cancer cells reduced the M2 polarization of both human CD14 ${ }^{+}$PBMCs and mouse TAMs. However, the overexpression of PKN2 with the K686R mutant, which abolished the ATP binding and reduced the catalytic activity of the protein $[23,28]$, significantly promoted the M2 polarization both in vitro and in vivo. These findings promoted the inhibition effect of PKN2 on the M2 polarization of TAMs.

Tumor cells secrete significant amounts of cytokines, such as IL4 and IL10, to promote M2 phenotype polarization in tumor microenvironment [8]. IL4 has 
been recognized as a potential tumor activator. Studies have shown that IL4, secreted by follicular helper T cells, downregulates antitumor immunity [29]. IL4 is also involved in radiation-induced aggressive tumor behavior in human cancer cells [30]. Clinical-based studies suggest that polymorphisms of IL4 and IL4R can affect susceptibility to gastrointestinal cancer [31]. Moreover, IL4 promotes M2 macrophage activation, which further induces cancer metastasis [32]. IL10 is an immunosuppressive cytokine that may facilitate carcinogenesis by downregulating interferon-gamma production and supporting tumor escape from the immune response. IL10 is significantly elevated in the serum and tumor microcirculation of patients with advanced stages of several cancers [32, 33]. In colorectal cancer, IL10 is mainly secreted by cancer cells, and polarizes TAMs to the M2 phenotype, which in turn promotes cancer cell migration and metastasis [6]. In the present study, we investigated the role of PKN2 in cytokines production in human colon cancer cells. We found that IL10 and IL4 were declined in PKN2-WT overexpressed cancer cells but elevated in PKN2-DN overexpressed cells. PKN2 inhibited the expression of IL10 and IL4 via regulating the transcription of the two cytokines. Furthermore, blocking IL10 and IL4 attenuated the upregulated M2 macrophages upon PKN2-depletion. Therefore, we concluded that PKN2 decreases the expression and secretion of IL10 and IL4 in colon cancer cells and eventually inhibits M2 macrophage polarization.

Mitogen-activated protein kinases (MAPKs) are a widely conserved family of serine/threonine protein kinases involved in many cellular programs, such as cell proliferation, differentiation, motility and death [34]. This family primarily comprises three kinase groups: the stress-activated protein kinases/c-Jun NH2-terminal kinases (Erk1/2), a second stress-activated MAPK group (p38 MAPKs) and a third class of stress-activated MAPK (Erk5) [35]. The present analysis of the KEGG pathway for gene expression profiling revealed a relation between PKN2 and MAPK pathways. Further study showed that PKN2 negatively regulates the phosphorylation of Erk1/2 but has no effect on p38 or Erk5. We further demonstrated that the MEK inhibitors partly blocked the elevated IL4 and IL10 in response to PKN2 knockdown. MEK inhibitors also reduced the M2 polarization of monocytes cocultured with PKN2 knockdown colon cancer cells. These results suggested that PKN2 contributed to the polarization of TAMs via regulating the Erk1/2-IL4/IL10 pathway.

Several TFs located downstream of Erk1/2, including Elk-1, FoxO3, CREB, Pax6 and STAT1/3 [36-39]. Among these TFs, Elk-1 and CREB was regulated by PKN2-Erk1/2 pathway, as assessed by our TFs activity assay. CREB is a phosphorylation dependent TF that stimulates transcription by binding to the DNA cAMP response element (CRE). CRE contains the highly conserved nucleotide sequence 5'-TGACGTCA-3', and CRE sites are typically found within the promoter or enhancer regions of genes. CREB is activated by Erk $1 / 2$ and induces several biological processes [39-41]. Elk-1 is a TF that binds to purine-rich DNA sequences. Elk-1 forms a ternary complex with SRF and the ETS and SRF motifs of the serum response element on the promoter region of genes. Elk-1 is a typical target of Erk1/2, and the Erk1/2-Elk-1 pathway participates in the expression of many genes, such as IL-1 $\beta$, collagen and TNF- $\alpha$ [42-44]. In the present study, we demonstrated that Elk-1 and CREB could be phosphorylated by Erk1/2, which mediated the expression of IL4 and IL10 by directly binding to their promoters. This process was suppressed by PKN2, explaining the mechanism by which PKN2 inhibited the expression of IL4 and IL10.

DUSP6 is a cytoplasmic MAP kinase phosphatase and expressed in a variety of tissues [45]. DUSP6 comprises a conserved C-terminal catalytic domain and an $\mathrm{N}$ terminal regulatory non-catalytic domain connected by a linker region. The linker region of DUSP6 contains important putative regulatory elements, including several residues subjected to phosphorylation, an active nuclear export sequence (NES) and a KIM-like motif [46-49]. Additionally, the linker region plays an important regulatory role [50, 51]. DUSP6 is an Erk1/2 specific phosphatase that specifically binds to and inactivates the Erk1/2 MAP kinases in mammalian cells [22]. We demonstrated that wild-type PKN2 elevated the phosphatase activity of DUSP6. Moreover, we uncovered the inner mechanism that PKN2 directly binds to the linker region of DUSP6 and promotes DUSP6 activity in Erk1/2 dephosphorylation.

The present study reports a new biological role for PKN2 in promoting the alternative activation of TAMs and inhibiting colon tumor growth. PKN2 reduced the polarization of TAMs towards the M2 phenotype by inhibiting Il10 and IL4 expression in colon cancer cells. PKN2 directly binds to DUSP6 to inactivate Erk1/2 and further suppress the transcriptional activities of CREB and Elk-1 by reducing their phosphorylation. Overall, we uncovered a novel role for PKN2 in the regulation of macrophage polarization and tumor growth in colon cancer (Fig. 7i). These findings suggest that targeting the PKN2 signaling pathway may be a potential therapeutic strategy for the treatment of colon cancer.

\section{Methods}

Collection of human colon cancer samples

Ninety samples from colon cancer patients who underwent surgery in Nanfang Hospital between June 2007 and April 2010 were obtained during operations. Seven 
polyp samples, 14 adenoma samples, 14 metastasis adenocarcinoma samples and ten normal colon tissue samples were collected. The diagnosis of cancer was confirmed by pathology. Patients with at least 5-year follow-up were included in this study. All specimens were acquired after signed informed consent using procedures approved by the Ethics Committee of Nanfang Hospital. This research was approved by the Ethics Committee of Nanfang Hospital. Tumor staging was determined according to AJCC Cancer Staging Manual. Patients' characteristics and histological data are shown in Table 2. A tissue microarray was constructed.

\section{Cell culture and transient transfection}

The human colon cancer cell line HCT116, RKO, SW480, HCT8 and HT-29 were obtained from Cell Bank of Typical Culture Preservation Commission, Chinese academy of Sciences. CD14 ${ }^{+}$monocytes were isolated from whole blood collected from healthy donors. HCT116 and HCT8 cells were cultured in RPMI1640 medium (Invitrogen), SW480 and HT-29 cells were cultured in DMEM (Invitrogen). Monocytes and macrophages were cultured in IMEM (Invitrogen). All medium was supplemented with $10 \%$ fetal bovine serum (Invitrogen) and $1 \%$ penicillin/streptomycin (Invitrogen). For transient transfection, cells were transfected with plasmid or siRNA using Lipofectamine 2000 and Opti-MEM (Invitrogen), according to the manufacturer's instructions. Sequences of the siRNAs are summarized in Additional file 1: Table S2.

\section{CD14 ${ }^{+}$peripheral blood mononuclear cells isolation}

The whole blood was collected from healthy donors. PBMCs were isolated by density gradient centrifugation using Ficoll-Paque ${ }^{\mathrm{Tw}}$ Plus (Amersham Pharmacia Biotech)

Table 2 Characteristics of patients with colon cancer

\begin{tabular}{lll}
\hline Characteristic & No.of patients & Percentage (\%) \\
\hline Gender & & \\
Male & 46 & 51.11 \\
$\quad$ Female & 44 & 48.89 \\
Age & & \\
$\quad \leq 55$ & 12 & 13.33 \\
$>55$ & 78 & 86.67 \\
Tumor location & & \\
$\quad$ Right Hemicolon & 40 & 44.44 \\
Left Hemicolon & 50 & 55.56 \\
Pathological type & & \\
Adenocarcinoma & 88 & 97.78 \\
Signet ring cell carcinoma & 2 & 2.22 \\
$\quad$ Undifferentiated carcinoma & 0 & 0.00 \\
\hline
\end{tabular}

according to the manufacturer's instructions. The diluted cellular fraction was overlaid onto the Ficoll-Paque Plus and subjected centrifugation at $900 \times \mathrm{g}$ for $30 \mathrm{~min}$. PBMCs were collected and washed twice with MACS $^{\circ}$ Buffer (Miltenyi Biotec $\mathrm{GmbH}$ ) by centrifugation at $450 \times \mathrm{g}$ for $10 \mathrm{~min}$. Immediately after collection, CD14 ${ }^{+}$ monocytes were isolated from PBMCs using a positive magnetic bead-assisted sorting assay (MC CD14 Monocyte Cocktail, human, Miltenyi Biotec $\mathrm{GmbH}$ ), according to the manufacturer's protocol. $\mathrm{CD} 14^{+}$monocytes purity was always above $95 \%$ as assessed by flow cytometry.

\section{Tumor xenograft study}

All protocols for animal research were approved by the Animal Care Committee of the Southern Medical University. Female nude BALB/c mice were raised in specific pathogen-free conditions with a 12-h light/dark schedule at $25{ }^{\circ} \mathrm{C}$. To generate subcutaneous mice colon cancer xenografts, $2.5 \times 10^{6} \mathrm{PKN} 2-\mathrm{WT} / \mathrm{PKN} 2-\mathrm{K} 686 \mathrm{R} /$ wild type control (WT) HCT116 cells were injected subcutaneously, respectively ( $n=10$ /group). Tumor size was measured once every other day using vernier caliper. Tumor volume was calculated based on two perpendicular measurements and using the formula: volume $=\left(\right.$ length $\times$ width $\left.^{2}\right) / 2$. Fifteen days after tumor cell inoculation, all mice were sacrificed, and the tumors were removed for further FACS, magnetic bead-assisted sorting assay and IHC analysis.

\section{Isolation of macrophages and tumor cells from mice tumor tissue}

Single cell suspensions were prepared from fresh tumors using Tumor Dissociation Kit (Miltenyi Biotec $\mathrm{GmbH})$. Cells were then immediately separated using a negative magnetic bead-assisted sorting assay (Mouse Cell Depletion Kit). TAMs were separated from the positive cell suspensions using $\mathrm{CD}_{11} \mathrm{~b}^{+}$magnetic beads. All operations were performed according to the manufacturer's protocol.

\section{Flow cytometry}

The cell suspension was collected and washed twice with MACS Buffer, and blocked by FcR Blocking Reagent (hu$\mathrm{man} /$ mouse) (Miltenyi Biotec $\mathrm{GmbH}$ ). Cells were then stained with CD206-FITC (human), CD206-PE (mouse), CD86-PE (human), CD16/32-APC.Cy7 (mouse), CD14APC (human) or CD11b-PE (mouse) antibodies (BD Biosciences, La Jolla, CA, USA), respectively. Separated mouse macrophages were permeated and fixed using Cytofix/CytoPerm Plus ${ }^{\mathrm{m}}$ kit (BD Biosciences) following the instruction, then stained with CD206 and CD16/32 antibodies. Cells were examined with a BD Accuri C6 flow cytometer (BD Biosciences) and all the tests were controlled by the homologous isotype control antibodies. 


\section{Immunohistochemistry}

The immunohistochemistry (IHC) staining was performed as described [52]. Microarray chips and mice xenografts were stained with anti-PKN2, anti-CD68, antiCD206, anti-CD86, anti-CD163, anti-CD16/32, antiDUSP6, anti-Ki67 (Abcam), anti-iNOS (Santa Cruz Biotechnology), and anti-phospho-Erk1/2 (Thr202/Tyr204), anti-Erk1/2 (Cell Signaling) antibodies. Control staining with only secondary antibodies was included to ensure specificity. Mouse IgG monoclonal-Isotype control and rabbit IgG polyclonal-Isotype control (Abcam, Cell Signaling \& Santa Cruz) were used as negative controls. Staining was independently assessed by two experienced pathologists (Wanfu Xu and Junhong Zhao) blinded to the clinical characteristics of the patients. The score for PKN2 and DUSP6 staining was based on the integrated staining intensity and the proportion of positive cells. Staining intensity was scored as follows: $0=$ no color; $1=$ yellow; $2=$ light brown; and $3=$ dark brown. The proportion of immune-positive tumor cells (number of positively labeled tumor cells / number of total tumor cells) was scored as follows: 0 , positive cells $<10 \% ; 1,10 \%-40 \%$ positive cells; $2,40 \%-70 \%$ positive cells; and 3 , positive cells $\geq 70 \%$. The final score was determined by adding the staining intensity score and average proportion of positive cells score and expressed as follows: 0 , negative staining, marked -; 0-2, weak expression, marked + ; 3-4, moderate expression, marked ++; and 5-6, strong expression, marked +++. IHC staining of CD68/iNOS/CD206 was calculated by the positive cell numbers in the stroma per high field. IHC staining of $\mathrm{p}$-Erk1/2 was calculated by the positive nuclear tumor cell numbers per high field. All the percentages/ numbers of positive cells were expressed as the average of six randomly selected microscopic fields.

\section{Luciferase assay}

Cells of $80 \%$ confluence were transfected using Lipofectamine 2000. Luciferase reporter gene plasmid and pRL-TK Renilla luciferase plasmid were co-transfected per well of a 12-well plate. Cell extracts were prepared at $22 \mathrm{~h}$ after transfection. The luciferase activity was measured with a Dual Luciferase Reporter Assay System (Promega).

\section{Co-immunoprecipitation (co-IP) assay}

Pretreated cells grown in $6 \mathrm{~cm}$ dishes were rinsed twice with precooled PBS, and lysed with $400 \mu$ l of ice-cold lysis buffer on ice for $30 \mathrm{~min}$, followed by centrifugation at $12000 \times \mathrm{g}$ for $10 \mathrm{~min}$. Supernatants were incubated with anti-DUSP6, anti-PKN2 (Abcam), anti-HA, antiflag (Cell Signaling) or control IgG at $4{ }^{\circ} \mathrm{C}$ overnight on a rotator, followed by addition of $30 \mu \mathrm{l}$ prewashed protein $\mathrm{A} / \mathrm{G}$ agarose beads for another $2 \mathrm{~h}$. After extensive washing with a diluted lysis buffer, the lysate was used for western blot analysis.

\section{Western blot analysis}

The cells were lysed, and proteins were extracted through standard protocols. The proteins were separated by SDS-polyacrylamide gel electrophoresis and subjected to western blot analyses. Protein bands were detected by the chemi-luminescence method. Specific primary antibodies against PKN2, GFP, DUSP6, p-Elk-1 (Ser383), Elk-1 (abcam), p-Erk1/2 (Thr202/Tyr204), Erk1/2, HA, flag, p-CREB (Ser133), CREB, Erk5, JNK1, JNK2, JNK3, p38, p-Erk5 (Thr218/Tyr220), p-JNK (Thr183/Tyr185), p-p38 (Thr180/Tyr182) (Cell Signaling) were used. GAPDH (Cell Signaling) was used as a loading control.

\section{RNA isolation, reverse transcription (RT) and real-time PCR}

Total RNA from tissues and cultured cell lines was isolated using the Trizol reagent (Invitrogen) according to the manufacturer's instruction. Primers for real-time RTPCR were designed using Primer Express v2.0 software (Applied BioSystems). Sequences of the primers are summarized in Additional file 1: Table S3. RT was carried out with the SuperScript First-Strand Synthesis System for RT-PCR (Invitrogen) according to the manufacturer's protocol. Real-time PCR was carried out using SYBR Green I (Applied BioSystems). The data were normalized to the geometric mean of housekeeping gene GAPDH and calculated as $2^{-\Delta \Delta C T}$.

\section{Cell counting kit-8 (CCK8) assay}

The pretreated cells were seeded into a 96-well plate. The cells were incubated with CCK8 reagent (DingGuo Bio) at $37{ }^{\circ} \mathrm{C}$ for $2 \mathrm{~h}$ and absorbance at $450 \mathrm{~nm}$ were measured using a microplate reader (BioTek).

\section{Cell cycle analysis}

Cells were detached using trypsinization, washed twice with precooled PBS, and fixed in $70 \%$ ethanol at $-20{ }^{\circ} \mathrm{C}$ overnight. The fixed cells were suspended in $100 \mu \mathrm{g} / \mathrm{ml}$ of RNaseA (KeyGen BioTECH) and $50 \mu \mathrm{g} / \mathrm{ml}$ of propidium iodide (PI) (KeyGen BioTECH) and incubated at room temperature for $40 \mathrm{~min}$ in the dark. After filtration, the cell cycle was examined by flow cytometry.

\section{Apoptosis assessment}

Following treatment, cells were washed with PBS and then stained using the Annexin V-FITC Apoptosis Detection Kit (Affymetrix eBioscience) according to the according the instruction. Cells were analyzed with a FACS flow cytometer (BD Biosciences).

\section{Gene expression profiles analysis}

Total RNA was isolated from pretreated cells with Trizol. RNA of HCT116 cells were labeled and hybridized to Human OneArray v7 (Phalanx Biotech Group) which contains 29,204 DNA oligonuceotide probes, and each 
probe is a 60-mer designed in the sense direction. Probes correspond to the annotated genes in RefSeq v42 and Ensembl v59 database. Signal intensity was normalized for each microarray and genes with a signal below 100 were ignored. Genes with a fold change equal to or higher than 2 compared with the control were picked out as potential targets.

\section{Plasmids and siRNA}

HA tagged plasmid PKN2-WT was constructed by ligating full-length open reading frame (ORF) of wild type PKN2 (1-936aa, Homo sapiens) and cloned into a expression vector pCMV-N-HA (Beyotime Biotech). PKN2-K686R plasmid was generated by PKN2-WT plasmid with a K686R point mutation at the ATP binding site. The expression vector pCMV-N-HA was used as control. The expression vector pCMV-N-flag (Beyotime Biotech) was used to construct plasmids that encode wild type DUSP6 with a N-terminal flag tag. pCMVDUSP6-WT expression plasmid was generated by ligating full-length open reading frame of DUSP6 (1-381aa, Homo sapiens) into pCMV-N-flag. The truncation mutant plasmids of DUSP6 were generated by ligating part of DUSP6's ORF into pCMV-N-flag (150-205aa; 1-205aa; 1-150, 205-381aa). The flag tagged plasmids containing full-length ORF of Elk-1 and CREB were purchased from Vigene Biosciences. The luciferase reporter plasmids were generated by ligating $-1500 \mathrm{bp} \sim 0 \mathrm{bp}$ of the promoter sequences of IL4 and IL1O into the pGL3ENHANCER plasmid (Promega Corp.). pRL-TK Vector was purchased from Promega.

\section{Lentivirus infection and stable clone selection}

The human shRNA sequences specifically targeting PKN2 (PKN2 shRNA\#1: 5' - CCGGTACTTTGGAAGT TCGTCTTATCTCGAGATAAGACGAACTTCCAGTA TTTTTG-3'; PKN2 shRNA\#2: 5'-CCGGGCAGGAA TTAAATGCACATATCTCGA.

GATATGTGCATTTAATTCCTGCTTTTT -3') were cloned into pGLVH1/ GFP + Puro vector (Genepharma). The expression construct of PKN2-WT (human) was generated by ligating full-length ORF of wild type PKN2 (1-936aa, Homo sapiens) and cloned into pGLV3/H1/ GFP + Puro vector (Genepharma). PKN2-K686R mutant (human) was created with a dominant negative $(\mathrm{DN})(\mathrm{K} 686 \mathrm{R})$ point mutation at the ATP binding site. Lentivirus was produced and collected after plasmid transfection of 293 T cells. HT-29 and SW480 cells were transduced with PKN2 shRNA or scramble shRNA (shCTL) lentivirus expressing GFP. SW480 and HCT116 cells were infected with PKN2-WT (human), PKN2K686R or control(Vector) lentivirus. Stable cell lines were selected by puromycin treatment $(2 \mu \mathrm{g} / \mathrm{ml})$ for
2 weeks. Knockdown or overexpression of PKN2 was confirmed by Western blotting.

\section{Soft agar assay}

$1 \times 10^{6}$ monocyte-derived macrophages were collected and seeded into the upper chamber of 24-well plates $(0.4 \mu \mathrm{m}$ pore size). Colon cancer cells were used for soft agar assay as previously described [53]. $1 \times 10^{4}$ HCT116 cells were cultured for 14 days. The medium was changed every $48 \mathrm{~h}$. Viable colonies larger than $50 \mu \mathrm{m}$ were counted.

\section{Macrophage and colon cancer cell co-culture}

After infected with shCTL/shPKN2-1/shPKN2-2, or vector/ PKN2-WT/ PKN2-K686R lentivirus, and selected the stably expression clones, the colon cancer cells were seeded into the upper chamber of 24-well plates $(0.4 \mu \mathrm{m}$ pore size) $\left(1 \sim 9 \times 10^{5}\right.$ cells/well) (Corning Corp., NY, USA). CD14 ${ }^{+}$monocytes were added in the lower chamber of the transwell apparatus according to the E:T ratio. Cells were co-cultured for indicated time and then harvested for subsequent experiment.

\section{Transcription factor activity array}

Nuclear extracts were prepared using Nuclear Extract Kit (Panomics, CA, USA) according to the manufacturer's instructions. The concentration of nuclear protein was determined using the bicinchoninic acid protein assay reagent kit (Pierce, Rockford, IL) to normalize for the amounts of protein within each experiment. TF array analysis (Panomics) was used to profile activities of 345 TFs. Any spots with a two-fold increase or decrease are considered significant.

\section{Chromatin immunoprecipitation (ChIP) assay}

ChIP analysis was performed on colon cancer cells transfected with pSuper, PKN2, or shPKN2 using the Pierce $^{\text {TM }}$ Magnetic ChIP Kit (Thermo Fisher) according to the manufacturer's instructions. PCR analysis were performed on immunoprecipitated DNA. After amplification, PCR products were separated on $1 \%$ agarose gels and visualized by ethidium bromide. Sequences of primers for promoter region used in this study are showed in Additional file 1: Table S4.

\section{ELISA}

ELISA was conducted according to the instructions. Concentrations of IL-4 (human), and IL-10 (human) in the culture supernatant of treated cells were measured with the use of a commercially available kit (CUSABIO). 


\section{Kinase activity assay}

Pretreated cells were dealed with immunoprecipitation assay as described above, and pulled down by antiDUSP6, anti-HA or anti-flag antibodies, respectively. PKN2 activity was determined with Universal Kinase Activity Kit (R\&D Systems) following the manufacturer's instruction. DUSP6 activity was determined with Phosphatase Assay kit (Sangon Biotech).

\section{Statistical analysis}

Data are presented as means \pm SEM from at least three experiments. All statistical analyses were performed using SPSS 13.0 (SPSS Inc.). Student's t test was used to compare control and treatment groups. The KaplanMeier estimation method was used for overall survival analysis, and a log-rank test was used to compare differences. $P<0.05$ was considered to be significant.

\section{Additional file}

Additional file 1: Table S1. KEGG pathway analysis of differentially expressed genes in colon cancer cells with different PKN2 expression level. Figure S1. The effect of M1-like and M2-like macrophages on proliferation of colon cancer cells in vitro. Figure S2. The effect of cardiolipin stimulation on cell proliferation and induction of macrophages differentiation of colon cancer cells. Figure S3. The effect of colon cancer cells on macrophages differentiation with different effector/target ratios in vitro. Figure S4. The effect of differently polarized macrophages on tumor cell proliferation. Figure S5. Heatmap of the gene expression array for cells with different PKN2 expression levels. Figure S6. The transcriptional factors involved in PKN2 mediated IL4 and IL10 expression. (DOC 34011 kb)

\section{Abbreviations}

CREB: Cyclic AMP-responsive element-binding protein; DUSP6: Dual specificity protein phosphatase 6; Elk-1: ETS domain-containing protein Elk-1; Erk: Extracellular signal-regulated kinase; IL: Interleukin; INOS: Inducible nitric oxide synthase; JNK: c-Jun N-terminal kinase; MAPK: Mitogen-activated protein kinase; p-Erk: Phosphorylation of Erk; PKC: Protein kinase C; PKN2: Protein kinase N2; RT-PCR: Reverse transcription-polymerase chain reaction; TAM: Tumor associated macrophage

\section{Acknowledgments}

We thank Dr. Yuxia Zhang for linguistic assistance during the preparation of this manuscript. We thank Dr. Jing Xie and Chaohui Xu for their technical support. We also thank Dr. Huan Chen, Lu Ren, Cuiping Liang and Huiwen Li for performing the data analysis.

\section{Funding}

This work was supported by Guangdong Natural Science Foundation [No. 2016A030310254], China Postdoctoral Science Foundation [No. 2016 M600648] and National Natural Science Foundation [No. 81602646] \& [No. 81301758].

\section{Availability of data and materials}

Data sharing not applicable to this article as no datasets were generated or. analyzed during the current study.

\section{Authors' contributions}

$Y C$ and $Y Z$ contribute equally to the work. LG and SG designed the study and drafted the manuscript. YC, YZ, JX, MY and PC prepared all the figures and Tables. WX and JZ reviewed the figures. All the authors reviewed and approved the final manuscript.

Ethics approval and consent to participate Not applicable.
Consent for publication

Not applicable.

\section{Competing interests}

The authors declare that they have no competing interests.

\section{Publisher's Note}

Springer Nature remains neutral with regard to jurisdictional claims in published maps and institutional affiliations.

\section{Author details}

'Digestive Department, Guangzhou Women and Children's Medical Center Guangzhou Medical University, No.9 Jinsui Road, Guangzhou, Guangdong 510623, China. ${ }^{2}$ Guangzhou Institute of Pediatrics, Guangzhou Women and Children's Medical Center, Guangzhou Medical University, Guangzhou, Guangdong 510623, China. ${ }^{3}$ Liver Tumor Center, Department of Infectious Diseases and Hepatology Unit, Nanfang Hospital, Southern Medical University, Guangzhou, Guangdong 510623, China.

Received: 3 July 2017 Accepted: 3 December 2017

Published online: 24 January 2018

References

1. Siegel R, Naishadham D, Jemal A. Cancer statistics, 2012. CA Cancer J Clin. 2012;62(1):10-29.

2. Lozano R, et al. Global and regional mortality from 235 causes of death for 20 age groups in 1990 and 2010: a systematic analysis for the global burden of disease study 2010. Lancet. 2012;380(9859):2095-128.

3. Biswas SK, Allavena P, Mantovani A. Tumor-associated macrophages: functional diversity, clinical significance, and open questions. Semin Immunopathol. 2013;35(5):585-600.

4. Ye Y, et al. Calcium influx blocked by SK\&F 96365 modulates the LPS plus IFN-gamma-induced inflammatory response in murine peritoneal macrophages. Int Immunopharmacol. 2012;12(2):384-93.

5. Xiao X, et al. M2 macrophages promote beta-cell proliferation by upregulation of SMAD7. Proc Natl Acad Sci U S A. 2014;111(13):E1211-20.

6. Zhang $Y$, et al. Crosstalk between colon cancer cells and macrophages via inflammatory mediators and CD47 promotes tumour cell migration. Eur J Cancer. 2013;49(15):3320-34.

7. Hu W, et al. Tumor-associated macrophages in cancers. Clin Transl Oncol. 2016;18(3):251-8.

8. Bingle L, Brown NJ, Lewis CE. The role of tumour-associated macrophages in tumour progression: implications for new anticancer therapies. J Pathol. 2002;196(3):254-65.

9. Jia W, et al. Galectin-3 accelerates M2 macrophage infiltration and angiogenesis in tumors. Am J Pathol. 2013;182(5):1821-31.

10. Na YR, et al. Cyclooxygenase-2 inhibition blocks M2 macrophage differentiation and suppresses metastasis in murine breast cancer model. PLoS One. 2013;8(5):e63451.

11. Zhang $Y$, et al. VDR status arbitrates the prometastatic effects of tumorassociated macrophages. Mol Cancer Res. 2014;12(8):1181-91.

12. Palmer RH, Ridden J, Parker PJ. Cloning and expression patterns of two members of a novel protein-kinase-C-related kinase family. Eur J Biochem. 1995;227(1-2):344-51.

13. Vincent S, Settleman J. The PRK2 kinase is a potential effector target of both rho and Rac GTPases and regulates actin cytoskeletal organization. Mol Cell Biol. 1997;17(4):2247-56.

14. Lee SJ, et al. PKN2 and Cdo interact to activate AKT and promote myoblast differentiation. Cell Death Dis. 2016;7(10):e2431.

15. Cryns VL, et al. Specific proteolysis of the kinase protein kinase C-related kinase 2 by caspase-3 during apoptosis. Identification by a novel, small pool expression cloning strategy. J Biol Chem. 1997;272(47):29449-53.

16. Koh $\mathrm{H}$, et al. Inhibition of Akt and its anti-apoptotic activities by tumor necrosis factor-induced protein kinase C-related kinase 2 (PRK2) cleavage. J Biol Chem. 2000;275(44):34451-8.

17. Schmidt $A$, et al. Rho GTPases regulate PRK2/PKN2 to control entry into mitosis and exit from cytokinesis. EMBO J. 2007;26(6):1624-36.

18. Lachmann S, et al. Regulatory domain selectivity in the cell-type specific PKN-dependence of cell migration. PLoS One. 2011;6(7):e21732. 
19. Yang CS, et al. The protein kinase $C$ super-family member PKN is regulated by mTOR and influences differentiation during prostate cancer progression. Prostate. 2017;77(15):1452-67.

20. Lin $\mathbf{W}$, et al. Protein kinase $C$ inhibitor chelerythrine selectively inhibits proliferation of triple-negative breast cancer cells. Sci Rep. 2017;7(1):2022.

21. Peng $B$, et al. Phosphorylation events associated with different states of activation of a hepatic cardiolipin/protease-activated protein kinase. Structural identity to the protein kinase N-type protein kinases. J Biol Chem. 1996;271(50):32233-40

22. Arkell RS, et al. DUSP6/MKP-3 inactivates ERK1/2 but fails to bind and inactivate ERK5. Cell Signal. 2008;20(5):836-43.

23. Gross C, Heumann R, Erdmann KS. The protein kinase C-related kinase PRK2 interacts with the protein tyrosine phosphatase PTP-BL via a novel PDZ domain binding motif. FEBS Lett. 2001;496(2-3):101-4.

24. Qian BZ, Pollard JW. Macrophage diversity enhances tumor progression and metastasis. Cell. 2010;141(1):39-51.

25. Chanmee T, et al. Tumor-associated macrophages as major players in the tumor microenvironment. Cancers (Basel). 2014;6(3):1670-90.

26. Komohara Y, et al. Clinical significance of CD163(+) tumor-associated macrophages in patients with adult T-cell leukemia/lymphoma. Cancer Sci. 2013;104(7):945-51.

27. Zhou X, Qi Y. Larynx carcinoma regulates tumor-associated macrophages through PLGF signaling. Sci Rep. 2015;5:10071.

28. Hodgkinson CP, Sale GJ. Regulation of both PDK1 and the phosphorylation of PKCzeta and -delta by a C-terminal PRK2 fragment. Biochemistry. 2002;41(2):561-9.

29. Shirota $\mathrm{H}$, et al. IL4 from T follicular helper cells Downregulates antitumor immunity. Cancer Immunol Res. 2017;5(1):61-71.

30. Kim ES, et al. IL-4, a direct target of miR-340/429, is involved in radiationinduced aggressive tumor behavior in human carcinoma cells. Oncotarget. 2016;7(52):86836-56

31. Cho YA, Kim J. Association of IL4, IL13, and IL4R polymorphisms with gastrointestinal cancer risk: a meta-analysis. J Epidemiol. 2017;27(5):215-20.

32. Wang J, et al. Novel mechanism of macrophage-mediated metastasis revealed in a zebrafish model of tumor development. Cancer Res. 2015;75(2):306-15.

33. Karlicic V, et al. Association of locally produced IL10 and TGFb1 with tumor size, histological type and presence of metastases in patients with lung carcinoma. J BUON. 2016:21(5):1210-8.

34. English JM, Cobb MH. Pharmacological inhibitors of MAPK pathways. Trends Pharmacol Sci. 2002;23(1):40-5.

35. Kyriakis JM, Avruch J. Mammalian mitogen-activated protein kinase signal transduction pathways activated by stress and inflammation. Physiol Rev. 2001;81(2):807-69.

36. Kortenjann M, Thomae $\mathrm{O}$, Shaw PE. Inhibition of v-raf-dependent c-fos expression and transformation by a kinase-defective mutant of the mitogen-activated protein kinase Erk2. Mol Cell Biol. 1994;14(7):4815-24.

37. Sunters $\mathrm{A}$, et al. Paclitaxel-induced nuclear translocation of FOXO3a in breast cancer cells is mediated by c-Jun $\mathrm{NH}_{2}$-terminal kinase and Akt. Cancer Res. 2006:66(1):212-20.

38. Yoo YD, et al. Fibroblast growth factor regulates human neuroectoderm specification through ERK1/2-PARP-1 pathway. Stem Cells. 2011;29(12):1975-82.

39. Wang J, et al. Chronic intermittent hypobaric hypoxia pretreatment ameliorates ischemia-induced cognitive dysfunction through activation of ERK1/2-CREB-BDNF pathway in anesthetized mice. Neurochem Res. 2017:42(2):501-12

40. Rajamanickam GD, Kastelic JP, Thundathil JC. The ubiquitous isoform of $\mathrm{Na}$ / K-ATPase (ATP1A1) regulates junctional proteins, connexin 43 and claudin 11 via Src-EGFR-ERK1/2-CREB pathway in rat Sertoli cellsdagger. Biol Reprod. 2017;96(2):456-68

41. Wang HJ, et al. IP-10/CXCR3 Axis promotes the proliferation of vascular smooth muscle cells through ERK1/2/CREB signaling pathway. Cell Biochem Biophys. 2017;75(1):139-47.

42. Larsen $\mathrm{CM}$, et al. Interleukin-1 beta-induced rat pancreatic islet nitric oxide synthesis requires both the p38 and extracellular signal-regulated kinase 1/2 mitogen-activated protein kinases. J Biol Chem. 1998;273(24):15294-300.

43. Bhogal RK, Bona CA. Regulatory effect of extracellular signal-regulated kinases (ERK) on type I collagen synthesis in human dermal fibroblasts stimulated by IL-4 and IL-13. Int Rev Immunol. 2008;27(6):472-96.

44. Tripathi A, Sodhi A. Growth hormone-induced production of cytokines in murine peritoneal macrophages in vitro: role of JAK/STAT, PI3K. PKC and MAP kinases Immunobiology. 2009;214(6):430-40.
45. Zhou B, et al. The specificity of extracellular signal-regulated kinase 2 dephosphorylation by protein phosphatases. J Biol Chem. 2002;277(35): 31818-25.

46. Bermudez $\mathrm{O}$, et al. Post-translational regulation of the ERK phosphatase DUSP6/MKP3 by the mTOR pathway. Oncogene. 2008;27(26):3685-91.

47. Karlsson $\mathrm{M}$, et al. Both nuclear-cytoplasmic shuttling of the dual specificity phosphatase MKP-3 and its ability to anchor MAP kinase in the cytoplasm are mediated by a conserved nuclear export signal. J Biol Chem. 2004:279(40):41882-91.

48. Marchetti $\mathrm{S}$, et al. Extracellular signal-regulated kinases phosphorylate mitogenactivated protein kinase phosphatase 3/DUSP6 at serines 159 and 197, two sites critical for its proteasomal degradation. Mol Cell Biol. 2005;25(2):854-64.

49. Zhou B, et al. Multiple regions of MAP kinase phosphatase 3 are involved in its recognition and activation by ERK2. J Biol Chem. 2001;276(9):6506-15.

50. Mark JK, et al. Inhibition of mitogen-activated protein kinase phosphatase 3 activity by interdomain binding. J Biol Chem. 2008;283(42):28574-83.

51. Cejudo-Marin R, et al. Caspase-3 cleavage of DUSP6/MKP3 at the interdomain region generates active MKP3 fragments that regulate ERK1/2 subcellular localization and function. J Mol Biol. 2012;420(1-2):128-38.

52. Cheng $Y$, et al. Synergistic anti-tumor efficacy of sorafenib and fluvastatin in hepatocellular carcinoma. Oncotarget. 2017:8(14):23265-76.

53. Zhu Y, et al. Protein kinase D2 contributes to TNF-alpha-induced epithelia mesenchymal transition and invasion via the PI3K/GSK-3beta/beta-catenin pathway in hepatocellular carcinoma. Oncotarget. 2015;7(5):5327.

\section{Submit your next manuscript to BioMed Central and we will help you at every step:}

- We accept pre-submission inquiries

- Our selector tool helps you to find the most relevant journal

- We provide round the clock customer support

- Convenient online submission

- Thorough peer review

- Inclusion in PubMed and all major indexing services

- Maximum visibility for your research

Submit your manuscript at www.biomedcentral.com/submit
) Biomed Central 